\title{
Acetabular Development Following Salter's Innominate Osteotomy for the Treatment of Developmental Dysplasia of the Hip: Long Term Follow-Up
}

\author{
Yalçin Turhan1, Bülent Kiliç², Murat Demiroğlu ${ }^{3 *}$, Esat Uygur, Korhan Özkan³, \\ Abdullah Eren ${ }^{5}$ \\ ${ }^{1}$ Department of Orthopaedics and Traumatology, School of Medicine, Duzce University, Duzce, Turkey \\ ${ }^{2}$ Department of Health Sciences, Gelisim University, Istanbul, Turkey \\ ${ }^{3}$ Department of Orthopaedics and Traumatology, School of Medicine, Medeniyet University, Istanbul, Turkey \\ ${ }^{4}$ Division of Orthopaedics and Traumatology, Emsey Hospital, Istanbul, Turkey \\ ${ }^{5}$ Department of Orthopaedics and Traumatology, School of Medicine, Istanbul Bilim University, Istanbul, \\ Turkey \\ Email: "drmuratdemiroglu@gmail.com
}

Received 16 March 2016; accepted 16 April 2016; published 19 April 2016

Copyright (C) 2016 by authors and Scientific Research Publishing Inc.

This work is licensed under the Creative Commons Attribution International License (CC BY).

http://creativecommons.org/licenses/by/4.0/

(c) (i) Open Access

\section{Abstract}

Aim: To investigate the long term results of the patients followed till the skeletal maturity and treated with Salter innominate osteotomy. Patients and Methods: 85 hips of 63 patients whom were operated by the same surgeon between the years 1985 and 1991 were evaluated retrospectively. 34 hips of 25 patients who did not have enough follow-up or did not reach skeletal maturity at their last radiographic control were excluded from the study. So, 51 hips of 38 patients were included into our study. Mean age of the patients at the time of operation was 3 years 2 months (1.5 11 years), mean follow-up time was 16 years 6 months (10 - 23 years) and mean age at the last control was 19 years 7 months (15 - 27 years). Results: The overall clinical results were favorable (excellent or good) in 48 hips $(\mathbf{9 4 . 1 \% )}$ ) according to the modified McKay clinical evaluation scale. Avascular necrosis was evaluated with the Kalamchi-McEven classification at the last follow up and 45 hips (88.2\%) had no avascular necrosis. The radiographic results were assessed by the Severin classification system and 10 hips (19.6\%) were stage $1 \mathrm{a} ; 10$ hips $(19.6 \%)$ were stage $1 \mathrm{~b}$; and 30 hips (58.8\%) were stage 2 . Upon the analysis of our radiographic results according to Stulberg classification system, we found out that only $2(3.9 \%)$ of our patients were Stulberg stage 5, whereas 36 cases $(70.6 \%)$ were Stulberg stage 1 and 2 (spherical congruency). According to Croft's classification of hips for degenerative changes, $36(70.6 \%)$ patients were stage 0 ; $6(11.8 \%)$ pa-

*Corresponding author. 
tients were stage $1 ; 5(9.8 \%)$ patients were stage 2 and 4 patients $(8.7 \%)$ were stage 3 . Conclusion: When appropriate soft tissue balance is provided, the complications of Salter Innominate Osteotomy are decreased and thus, it provides an excellent functional and radiological result.

\section{Keywords}

\section{DDH, Salter, Innominate Osteotomy, Avascular Necrosis, Remodeling}

\section{Introduction}

Developmental dysplasia of the hip (DDH) encompasses a wide range of hip development disorders which arise in different age groups, due to a variety of underlying processes. As DDH primarily affects young children, treatment options vary considerably based upon the age of the patient at the time of diagnosis. Regardless of the underlying cause, the primary goal of DDH treatment is to achieve a stable, anatomical and concentric reduction of the hip resulting in a clinically sufficient improvement in joint function. Closed or open reductions are often sufficient for patients under the age of 18 months, with surgical alteration of the proximal femur and acetabulum necessary for children of walking age; however, the age at which the remodeling capacity of the acetabulum is the most compatible with DDH treatment remains poorly understood [1].

Salter's innominate osteotomy is a surgical intervention first described in 1961 for patients with late diagnosis or insufficient remodeling after the initial treatment [2]. In this procedure, stability of the reduced hip is achieved via redirection of the acetabulum, in which the weight-bearing area of the acetabulum is increased without a change in volume or shape [3]. However, despite the long history of this procedure, little is known regarding the long term outcomes of patients once their skeletal development has been completed. Here, we sought to evaluate the long-term effects of preoperative traction, open reduction, capsulorrhaphy, and Salter's innominate osteotomy on acetabular development in patients 18 months and older treated for DDH between 1985 and 1991.

\section{Patients and Methods}

Salter's innominate osteotomy was performed as treatment for DDH by the senior author (A.E.) on 85 hips across 63 individuals between 1985 and 1991. From this initial group, we performed a retrospective analysis of all patients with at least 10 years of follow-up who had completed their skeletal development. A total of 25 patients (34 hips) were excluded from this study due to either insufficient follow-up or insufficient skeletal development at the time of the study, as determined based upon data from their last follow-up, resulting in a final study population of 51 hips across 38 patients. Of these patients, $6(15.7 \%)$ were male and $32(84.3 \%)$ were female. Bilateral involvement was seen in $13(34.2 \%)$ patients; $11(28.9 \%)$ patients underwent surgery for their right hip, and 14 (36.8\%) for their left hip (Figure 1 and Figure 2).

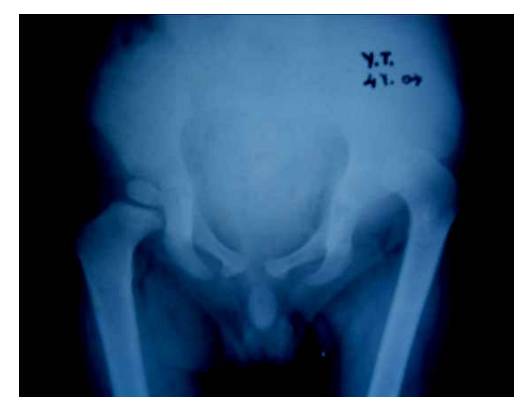

(a)

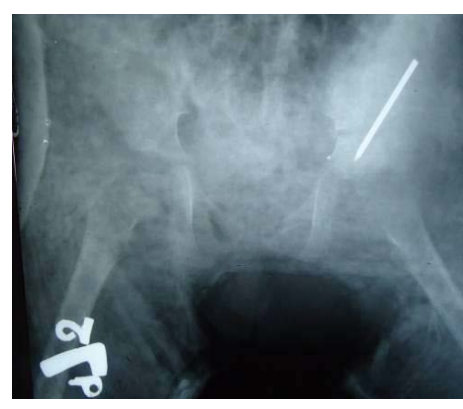

(b)

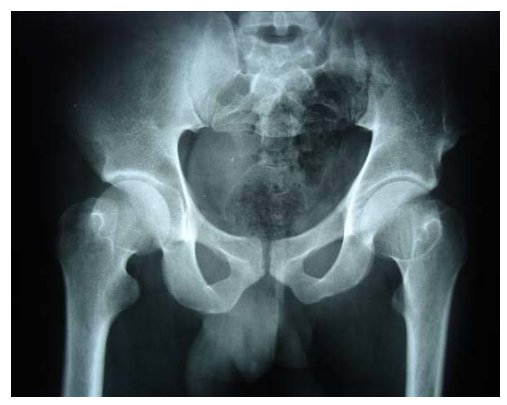

(c)

Figure 1. (a) Male patient 4 years of age with left hip high-riding dislocation (Tönnis grade IV). Plain X-ray, anteroposterior (AP) view; (b) Plain X-ray, early postoperative time point, AP view; (c) Plain X-ray, 23-year postoperative time point, AP view, with excellent clinical and radiographic results. 


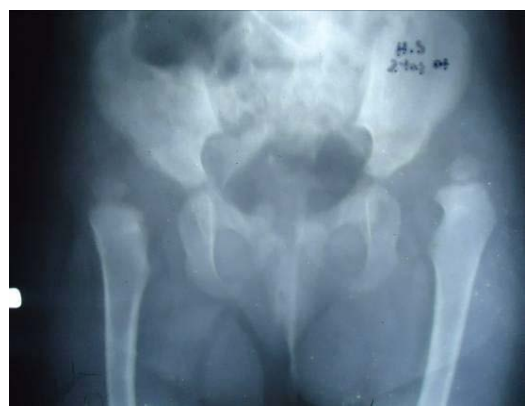

(a)

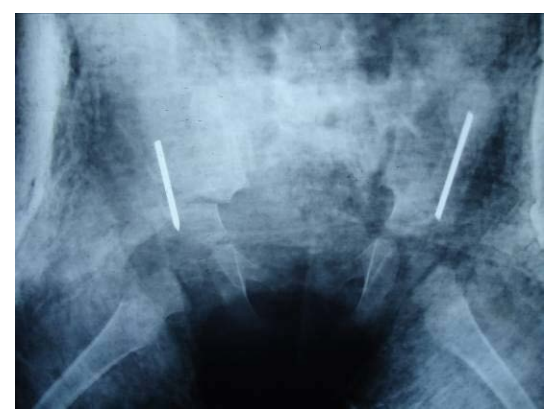

(c)

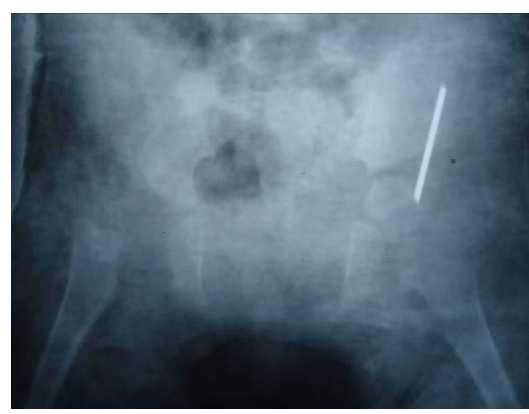

(b)

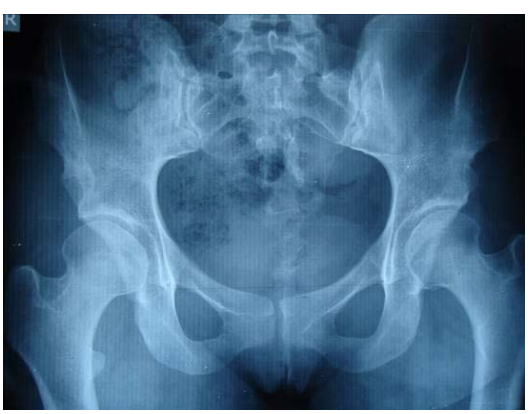

(d)

Figure 2. (a) Female patient 2 years of age with bilateral Tonnis type II dislocation. Plain X-ray, AP view; (b) Plain X-ray, early postoperative time point, AP view, left side; (c) Plain X-ray, early postoperative time point, AP view, right side (Surgery of the right side was performed at the same time as removal of the left spica cast); (d) Plain X-ray, 22-year postoperative time point, AP view, with excellent clinical and radiographic results except for the left hip; radiographically the Stulberg and Severin grades were 3 and 2, respectively.

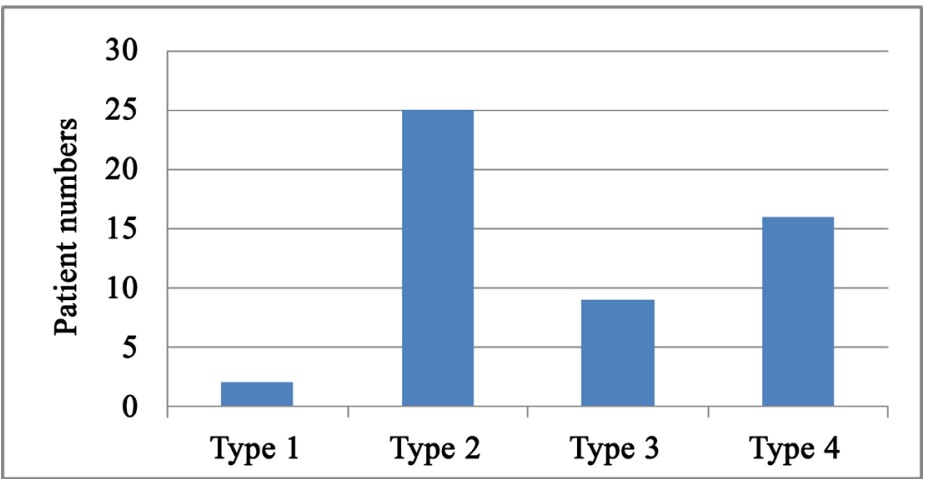

Figure 3. Distribution of patients' hips according to Tönnis types.

The patients' age at operation varied between 1.5 and 11 years, with a mean age of 3 years, 2 months. The follow-up duration ranged from 10 to 23 years, with a mean duration of 16 years, 6 months. Mean age at the time of their most recent follow-up was 19 years, 7 months, with a range of 15 to 27 years. Of the 51 hips included in the study, 1 was Tönnis type $1(2 \%) ; 25$ were Tönnis type $2(49 \%)$; 9 were Tönnis type $3(17 \%)$; and 16 were Tönnis type 4 (31.4\%) (Figure 3 ).

Comorbidities were observed in two male patients; one had multiple skeletal system deformities, and the other had bilateral talipes equinovarus deformity; no significant co-morbidities were observed in any other patients. Limping and shortness of one leg were the most common complaints in patients with unilateral disease, while swaying while walking (waddling gait) was more commonly reported in cases of bilateral disease.

Preoperatively, patients under the age 3 (25 hips) underwent 2 weeks of skin traction, whereas patients 3 years of age and older (25 hips) underwent 2 weeks of skeletal traction. Skeletal traction was applied for 1 week to the left hip of a 5-year-old patient who had bilateral involvement. All the patients received general anesthesia during 
surgery, with the procedure taking place on a general purpose orthopedic operation table.

Salter's osteotomy was performed according to the technique described by Salter and Dubos in 1961. Postoperatively, a pelvipedal cast was applied down to the knee, with the operated hip held at $10-20$ degrees flexion, 20 - 30 degrees abduction, the knee at $10-20$ flexion, and the ankle at neutral position; the other extremity was positioned with the hip at the same position. No intraoperative complications were reported for any patient. Casts were removed 6 weeks after treatment, and the patient was fitted with a Dennis-Brown abduction brace, which was to be worn 24 hours a day for 3 months, followed by nights only for the next 3 months, for a total of 6 months.

Acetabular indices (AI) of all patients were evaluated by Hilgenreiner's method (the angle between the Y-line -connecting the lowest points of the ilium and the line which connects the lowest point of the ilium and the acetabular edge) [4] and Tönnis classifications made using a preoperative pelvis graphy. AI was measured with a pelvis graphy in the early postoperative period. On the last follow-up, Sharp's AI angle, Wilberg's center edge (CE) angle, femoral head coverage ratio, head trochanter relation, and Tönnis AI angle were evaluated through roentgenography. Avascular necrosis (AVN) class was evaluated using the Kalamchi-McEven classification [5], and the state of hip arthrosis was evaluated according to Croft's classification [6], femoral head-acetabulum congruence was evaluated in accordance with Stulberg staging [7], and radiographic results were evaluated using the Severin classification [8].

\section{Statistical Analyses}

We use NCSS (Number Cruncher Statistical System) 2007 Statistical Software (Utah, USA) pocket program for all statistical analyses. "Tukey's multiple comparison test" was used for subgroup comparisons, "independent t test" was used for pair comparisons, "Chi-squared test" was used for qualitative data and "Pearson correlation test" was used for relationship between variables. Significance statements refer to P values of two-tailed tests that were $<0.05$.

\section{Results}

Evaluation of radiographies revealed a mean preoperative acetabular index of $38.3^{\circ}$ (range: $17^{\circ}$ and $50^{\circ}$ ), compared with a mean early postoperative acetabular index of $19.2^{\circ}$ (range: $10^{\circ}$ and $30^{\circ}$ ). The mean Sharp angle on the last follow-up graphy was $40.2^{\circ}$ (range: $38^{\circ}$ and $48^{\circ}$ ), while the mean CE angle was $29.1^{\circ}$ (range: $15^{\circ}$ and $\left.50^{\circ}\right)$.

In the last follow-up graphy, when the avascular necrosis was evaluated, 45 hips (88.2\%) had no AVN, 2 hips (3.9\%) had grade 1; 3 hips (5.9\%) had grade 2; and 1 hip (2\%) had grade 4 AVN (Figure 4). A second operation, femoral shortening, was performed on the patient who had grade $4 \mathrm{AVN}$ due to insufficient preoperative traction along with joint stiffness and abduction contracture.

The mean femoral head coverage ratio was $84.9 \%$ (range: $70 \%-110 \%$ ). The femoral head-trochanter relationship was neutral in 18 hips (35.3\%), negative in 19 hips (37.3) and positive in 14 hips (27.5\%). According to Croft's classification, 36 hips (70\%) were stage $0 ; 6$ hips (12\%) were stage 1; 5 hips (10\%) were stage 2 ; and 4

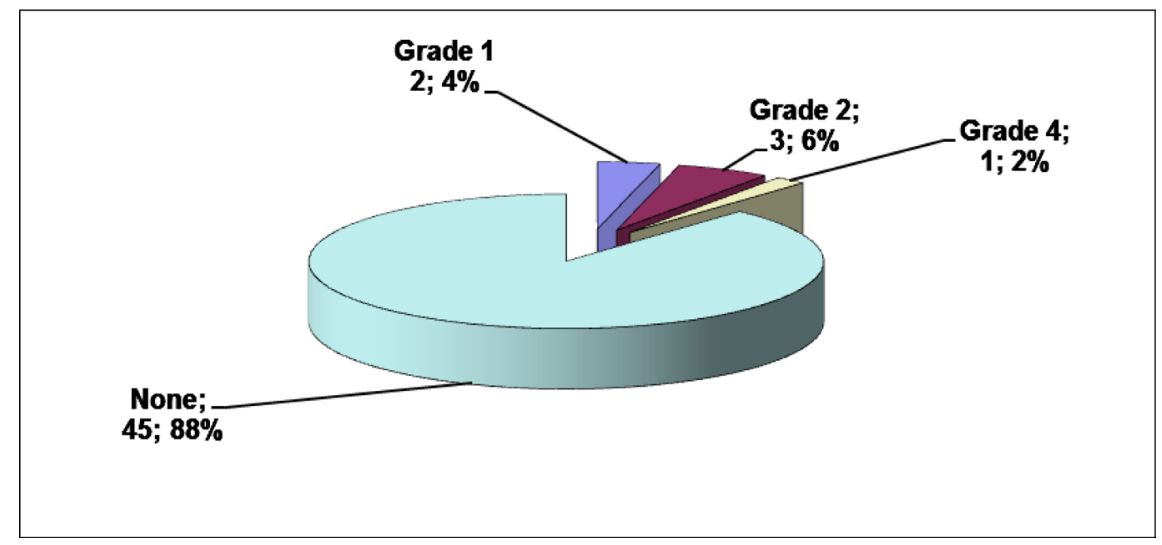

Figure 4. AVN grade distribution of the hips. 
hips (8\%) were stage 3. Using Stulberg's classification, 20 hips (39\%) were stage 1; 16 hips (31\%) were stage 2; 10 hips (20\%) were stage 3; 3 hips (6\%) were stage 4; and 2 hips (4\%) were stage 5 (Figure 5).

Evaluations using the modified McKay clinical evaluation scale revealed 28 hips (54.9\%) classified as group 1,20 hips $(39.2 \%)$ as group 2 , and 3 hips $(5.8 \%)$ as group 3 . The mean Tönnis acetabular index was $10.4^{\circ}$ (range: $\left.8^{\circ}-20^{\circ}\right)$ in the most recent follow-up graphy. According to the Severin classification system, 10 hips $(19.6 \%)$ were stage 1a, 10 hips (19.6\%) were stage 1b, 30 hips (58.8\%) were stage 2, and 1 hip (2\%) was stage 3 (Figure 6).

No statistically significant relation was observed between the age at operation and Croft's classification stage $(\mathrm{P}=0.497)$; however, age was significantly associated with both Stulberg and Severin stage classifications. The mean age of Stulberg stage 4 patients was significantly higher relative to other stages, with no statistically significant differences observed between other Stulberg stages. The overall association between Severin stage and mean age was statistically significant $(\mathrm{P}=0.048)$, with the mean age of stage 2 patients significantly higher than that of stage 1 at the time of operation $(\mathrm{P}=0.042)$; no statistically significant relation was found between other stages $(\mathrm{P}>0.05)$.

No statistically significant difference was found between Tönnis stage distribution and AVN development (P $=0.698)$, Croft's classification $(\mathrm{P}=0.527)$, or Severin stage $(\mathrm{P}=0.536)$. A statistically significant positive relationship was found between the preoperative AI values and follow-up AI values $(r=0.296 ; \mathrm{P}=0.048)$. Patients with higher preoperative AI values were found to have higher follow-up Tönnis AI values. No statistically significant difference was found between the age at operation and AVN development. $(\mathrm{P}=0.863)$.

\section{Discussion}

Over the past two decades, early detection of hip dysplasia has become possible, even in newborns, due to the widespread use of ultrasonography, minimizing the need for medial open reductions. This, in turn, has led to a decrease in technical proficiency among surgeons in developed countries regarding the treatment of this disorder,

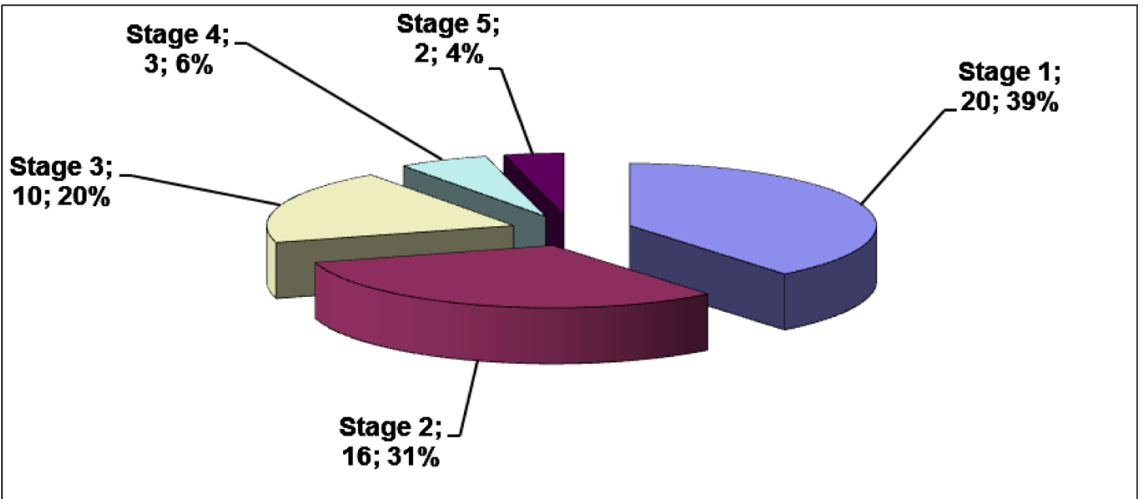

Figure 5. Results of Stulberg's classification of the hips.

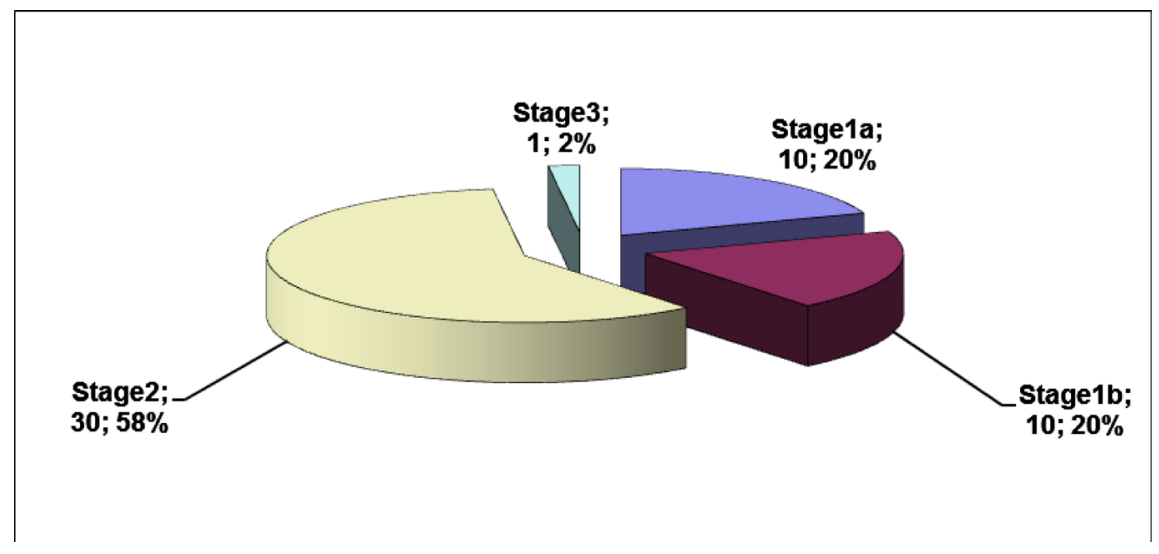

Figure 6. Severin classification results of the hips. 
outside of a small number of specialized institutions. On the other hand, DDH is still is a major orthopedic problem in developing countries, where orthopedic surgeons frequently encounter such cases. Here, we sought to evaluate the long-term effects of preoperative traction, open reduction, capsulorrhaphy, and Salter's innominate osteotomy on acetabular development in patients 18 months and older treated for DDH between 1985 and 1991.

Although closed or open reduction alone is usually sufficient in infants younger than 18 months of age, preoperative traction, open reduction, and pelvic $+/-$ femoral rotational or shortening osteotomies are often necessary in children of walking age due to secondary changes in the femoral head and acetabulum, along with the decreased remodeling capacity of these structures over time [2].

Salter's innominate osteotomy, first described in 1961, reorients the acetabulum to produce a larger safe zone within the hip joint, thereby ensuring normal development. In this procedure, the symphisis pubis serves as a hinge on which the acetabulum, including the pubis and the ischium, rotates as a unit [2].

Open reduction and pelvic osteotomy used for the treatment of hip dysplasia is a technically difficult procedure. Reorientation of the acetabulum becomes even more difficult after open reduction, during which the anterior capsule is incised. Therefore, some authors have suggested a two-stage surgery consisting of an open reduction followed by Salter's osteotomy. However, this approach comes with a serious disadvantage since two separate surgeries and two casting periods are required, thereby doubling the overall rehabilitation time. Furthermore, scar tissue complicates the second surgery, and treatment costs are increased [2].

In this study, we relied on the Severin classification system to evaluate radiographic results. This classification system is an ideal method as it offers high inter-observer reliability, and is able to reflect long-term radiological and functional outcomes [9]. Using this classification system, we obtained good to excellent results in $98 \%$ of our cases treated with open reduction and Salter's osteotomy, a modest improvement compared to previous reports. In similar studies, Gülman et al. reported $71.1 \%$ good to excellent results in 52 hips from 39 patients, with a mean follow-up of 13 years [10]. Haider et al. reported $83.8 \%$ good to excellent radiographic results with a mean follow-up of 91 months [3], while Salter and Dubos reported 93.6\% good to excellent results according to Severin classification in patients under 4 years of age [11].

Stage 1 or 2 avascular necrosis was detected in $9.8 \%$ of all cases, with a total avascular necrosis rate of $11.8 \%$. In one case, which was complicated by stage 4 avascular necrosis, preoperative traction was applied for only 1 week and a secondary femoral shortening and derotation osteotomy had to be performed due to the occurrence of an abduction contracture 6 months after completion of the second stage of surgery. This occurrence is consistent with the results of Gage and Winter, who reported a decrease in avascular necrosis with preoperative traction [3].

Stulberg et al. devised a classification system with 5 sub-classes to predict the long-term prognosis after Perthes disease and to estimate the time at which degenerative joint disease would appear [12]. Although Neyt et al. showed skepticism about the usability and reliability of this classification system due to high inter-observer variability [12], excellent inter- and intra-observer reliability has been reported for this system [13] [14]. While the Stulberg classification system was originally devised to predict long-term outcome in Perthes disease, Shinoda et al. has since expanded the use of this classification system to include outcomes of periacetabular osteotomies in the treatment of dysplastic hips [15]. Using this system, we observed only $2(3.9 \%)$ patients classified as Stulberg stage 5, with the vast majority (36 cases, 70.6\%) classified as either stage 1 or 2 (spherical congruency), indicative of a good prognosis. Given the small number of Stulberg stage 5 patients, these two patients were excluded from our statistical analyses. Despite this exclusion, Stulberg stage 4 patients were found to be significantly older at the time of operation relative to lower stages, likely the result of secondary changes in the hip joints of older children.

Croft's classification system was used to assess degenerative changes due to hip dysplasia [16]. According to this system, $36(70.6 \%)$ patients were classified as stage $0 ; 6(11.8 \%)$ patients were stage $1 ; 5(9.8 \%)$ patients were stage 2 ; and 4 patients $(8.7 \%)$ were stage 3 ; No patients were designated as stage 4 or 5 . The high percentage of stage 0 patients in this analysis is consistent with the high number of stage 1 and 2 patients using the Stulberg system, indicating a high degree of overlap between methodologies.

One of the most important factors to take into account when evaluating clinical outcomes is the age of the patient at the time of operation. Salter et al. recommended the use of innominate osteotomy in patients 18 months to 6 years of age, though success rates varied considerably with age, falling from $93.6 \%$ in patients 1.5 - 3 years of age to only $56.7 \%$ in $4-6$ year olds [11]. Similar results were also reported by McCay, et al. with good to 
excellent outcomes seen in $90 \%$ of patients 1.5 - 4 years of age, with success rates falling to $68.5 \%$ in patients 5 - 7 years of age, and $56 \%$ in patients 8 years of age and older. A third study by Kapukaya et al. reported well to excellent results in $95.4 \%$ of patients 1.5 - 3 years of age, $72.2 \%$ in patients $4-6$ years of age, and $62.5 \%$ in 7 10 year olds [17]. Here, we observed good to excellent results in all patients 5 years of age or older at the time of operation. This unusually high success rate is likely due to a combination of factors, including effective and meticulous preoperative traction, the same highly experienced surgeon performing all surgeries, capsuloplasty, minimal soft tissue dissection, and the effectiveness of Salter's osteotomy with continuing remodelization capacity.

Within our patient cohort, we observed a statistically significant increase in the number of Severin class 2 patients as compared to Severin class 1, which was strongly correlated with patient age at the time of operation. This association is likely the result of secondary osseous and soft tissue changes around the hip joint in older patients. Tönnis was developed a grading system according to the position of the proximal femoral epiphysis on the AP roentgenography [18]. According to this classification system; the proximal femoral epiphysis is medial to the Perkin's line in grade 1, lateral to the Perkin's line but below to the horizontal line traversing the upper lateral border of the acetabulum in grade 2, at the level of this horizontal line in grade 3 and superior to horizontal line in grade 4 . We did not observe any statistically significant differences associated with risk of avascular necrosis, patient's age at the time of operation, or preoperative Tönnis stage relative to published results.

A post-osteotomy decrease in posterior coverage remains a clear disadvantage of Salter's osteotomy, though acetabular development anomalies due to developmental dysplasia of the hip have also been shown to result in anterolateral insufficiency [19]. While, it has been suggested that Salter's osteotomy causes triradiate cartilage to close early due to excessive scraping of the iliac bone around acetabular physis, this observation has yet to be replicated [20].

Femoral head anteversion remains an important consideration in DDH. Salter's osteotomy allows for sufficient femoral head coverage, particularly when preoperative traction is applied, eliminating the need for femoral derotation. In addition, after Salter's innominate osteotomy, the posterior coverage defect has never been large enough to negatively affect hip stability, as defined by either radiological or functional outcomes. We believe these results are due in large part to our use of preoperative traction, which reduces the amount of pressure put on the femoral head as a result of Salter's osteotomy, thereby preventing many of the complications which may arise due to this procedure.

\section{Conclusion}

Salter's innominate osteotomy has been a widely successful surgical technique for the treatment of DDH. Proper application of traction and other methods aimed at reducing the stress placed on soft tissues strongly reduces the complication rate associated with this procedure, resulting in excellent long term functional and radiological outcomes. Further studies comparing the results of treatment of DDH either with preoperative traction or femoral shortening osteotomy may be needed for evaluation of the optimal surgical benefit.

\section{Informed Consent Statement}

All study participants, or their legal guardian, provided informed written consent prior to study enrollment.

\section{Institutional Review Board Statement}

This study was reviewed and approved by the Goztepe Training and Research Hospital Institutional Review Board.

\section{Conflict of Interest}

The authors declared no conflicts of interest with respect to the authorship and/or publication of this article.

\section{References}

[1] Herring, J.A. (2002) Developmental Dysplasia of the Hip. In: Herring, J.A., Ed., Tachdjian's Pediatric Orthopaedics, Vol. 1, 3rd Edition, WB Saunders, Philadelphia, 513-654.

[2] Salter, R.B. (1961) Innominate Osteotomy in the Treatment of Congenital Dislocation and Subluxation of the Hip. The Journal of Bone \& Joint Surgery, 43, 518-539. 
[3] Haidar, R.K. and Jones, R.S. (1996) Simultaneous Open Reduction and Salter Innominate Osteotomy for DDH. The Journal of Bone \& Joint Surgery, 78, 471-476.

[4] Hilgenreiner, H. (1925) Early Diagnosis and Early Treatment of Congenital Dislocation of the Hip. Medizinische Kli$n i k, 21,1385-1429$.

[5] Kalamchi, A. and MacEwen, G.D. (1980) Avascular Necrosis Following Treatment of Congenital Dislocation of the Hip. Journal of Bone \& Joint Surgery, 62, 876-888.

[6] Croft, P., Cooper, C., Wickham, C. and Coggon, D. (1990) Defining Osteoarthritis of the Hip for Epidemiologic Studies. American Journal of Epidemiology, 132, 514-522.

[7] Stulberg, S.D., Cooperman, D.R. and Wallensten, R. (1981) The Natural History of Legg-Calv'e-Perthes Disease. Journal of Bone \& Joint Surgery, 63, 1095-1108.

[8] Severin, E. (1941) Contribution to the Knowledge of Congenital Dislocation of the Hip Joint. Late Results of Closed Reduction and Arthrographic Studies of Recent Cases. Acta Chirurgica Scandinavica, 84, 1-16.

[9] Varner, K.E., Incavo, S.J., Haynes, R.J. and Dickson, J.A. (2010) Surgical Treatment of Developmental Hip Dislocation in Children Aged 1 to 3 Years: A Mean 18-Year, 9-Month Follow-Up Study. Orthopedics, 33, 162. http://dx.doi.org/10.3928/01477447-20100129-05

[10] Gülman, B., Tuncay, I.C., Dabak, N. and Karaismailoğlu, N. (1994) Salter's Innominate Osteotomy in the Treatment of Congenital Hip Dislocation: A Long Term Review. Journal of Pediatric Orthopaedics, 14, 662-666. http://dx.doi.org/10.1097/01241398-199409000-00021

[11] Salter, R.B. and Dubos, J.P. (1974) The First Fifteen Years Personal Experience with Innominate Osteotomy in the Treatment of Congenital Dislocation and Subluxation of the Hip. Clinical Orthopaedics, 98, 72. http://dx.doi.org/10.1097/00003086-197401000-00009

[12] Neyt, J.G., Weinstein, S.L., Spratt, K.F., Dolan, L., Morcuende, J., Dietz, F.R., Guyton, G., Hart, R., Kraut, M.S., Lervick, G., Pardubsky, P. and Saterbak, A. (1999) Stulberg Classification System for Evaluation of Legg-Calvé-Perthes Disease: Intra-Rater and Inter-Rater Reliability. Journal of Bone \& Joint Surgery, 81, 1209-1216.

[13] Agus, H., Kalenderer, Ö., Eryanlmaz, G. and Ozcalabi, İ.T. (2004) Intraobserver and Interobserver Reliability of Catterall, Herring, Salter-Thompson and Stulberg Classification Systems in Perthes Disease. Journal of Pediatric Orthopaedics $B, 13,166-169$.

[14] Farsetti, P., Tudisco, C., Caterini, R., Potenza, V. and Ippolito, E. (1995) The Herring Lateral Pillar Classification for Prognosis in Perthes Disease. Late Results in 49 Patients Treated Conservatively. Journal of Bone \& Joint Surgery, 77, 739-742.

[15] Tsuyoshi, S., Masatoshi, N. and Yoshinari, N. (2009) Periacetabular Osteotomy for the Treatment of Dysplastic Hip with Perthes-Like Deformities. International Orthopaedics (SICOT), 33, 71-75. http://dx.doi.org/10.1007/s00264-007-0476-9

[16] Steffen, J., Stig, S.H., Kjeld, S. and Peter, G. (2005) Hip Dysplasia and Osteoartrosis. Acta Arthopaedica, 76, $149-158$.

[17] Berkeley, M.E., Dickson, J.H. and Cain, T.E. (1984) Surgical Therapy for Congenital Dislocation of the Hip in Patients Who Are Twelve to Thirty-Six Months Old. Journal of Bone \& Joint Surgery, 66, 412-420.

[18] Tönnis, D. (1987) Clinical and Radiographic Schemes for Evaluating Therapeutic Results. In: Tönnis, D., Ed., Congenital Dysplasia and Dislocation of the Hip in Children and Adults, Springer, Heidelberg, 165-171. http://dx.doi.org/10.1007/978-3-642-71038-4 13

[19] Takashi, S., Hattori, T., Konishi, N. and Iwata, H. (1998) Acetabular Development after Salter's Innominate Osteotomy for Congenital Dislocation of the Hip: Evaluation by Three-Dimensional Quantitative Method. Journal of Pediatric Orthopaedics, 18, 802-806. http://dx.doi.org/10.1097/01241398-199811000-00020

[20] Makin, M. (1980) Closure of the Epiphysis of the Femoral Head and of the Triradiate Cartilage of the Acetabulum Following Surgery for Congenital Hip. Israel Journal of Medical Sciences, 16, 307-310. 